\title{
A structural health monitoring of a pitch catch active sensing of PZT sensor on normal, damage and repair aircraft spoiler
}

\begin{abstract}
At present, aircraft structural integrity is a concerned due to heavy usage of composite materials and cost saving on the operational. Structural health monitoring system is one of condition based monitoring introduced to supplement the current aircraft maintenance non destructive inspection. One approach is to embed or attach sensors such as lead zirconate titanate (PZT) to detect the anomalies either passively or actively. Due to the aircraft operational environment the defects and damage are likely to occur. Repair has to be carried out as per recommended and the requirement to replace back the sensors are important in order to monitor back the structure at post repair situation. The Lamb waves generated by using the PZT sensor can be used to monitor the surface structural integrity for damage or pristine condition. The effect of the lamb wave signals when surface condition of the aircraft component changes is concerned. One hundred data sets were recorded for the undamaged, damage and repair condition. An outlier analysis was used to analyze the situation by overlaying the isolated signal spectrum and the range of the voltage peak to peak (Vpp) mean values. Different signals were observed for different type of structural condition tested and more tests were required to make a conclusive solution.
\end{abstract}

Keyword: Composite repair; Lead zirconate titate (PZT) sensors and outlier analysis; Structural health monitoring system 